\title{
Audiometric thresholds and prevalence of tinnitus among male veterans in the United States: Data from the National Health and Nutrition Examination Survey, 1999-2006
}

\author{
Robert L. Folmer, PhD; ${ }^{1-2 *}$ Garnett P. McMillan, PhD; ${ }^{1,3}$ Donald F. Austin, MD, MPH; ${ }^{1,3}$ James A. Henry, PhD ${ }^{1-2}$ \\ ${ }^{1}$ National Center for Rehabilitative Auditory Research, Portland Department of Veterans Affairs Medical Center, \\ Portland, OR; Departments of ${ }^{2}$ Otolaryngology and ${ }^{3}$ Public Health and Preventive Medicine, Oregon Health \& \\ Science University, Portland, OR
}

\begin{abstract}
Hearing loss and tinnitus are the two most prevalent service-connected disabilities among U.S. veterans. The number of veterans receiving compensation and services from the Department of Veterans Affairs (VA) for these conditions continues to increase annually. However, the majority of veterans in the United States do not use VA medical centers or clinics for healthcare and do not receive VA compensation payments. Therefore, the prevalence of hearing loss and tinnitus among U.S. veterans is unknown. This study used National Health and Nutrition Examination Survey data to estimate the prevalence of these auditory conditions among male veterans. Between 1999 and 2006, pure tone audiometric data collected from 845 male veterans were compared with pure tone thresholds collected from 2,086 male nonveterans. We used questionnaire data collected between 1999 and 2004 to calculate and compare the prevalence of tinnitus for 2,174 veterans and 4,995 nonveterans. In general, pure tone thresholds did not differ significantly between veterans and nonveterans for most frequencies tested $(500-8,000 \mathrm{~Hz})$. The overall prevalence of tinnitus was greater for veterans than that for nonveterans $(p<$ 0.001 ), with statistically significant differences in the 50 to 59 and 60 to 69 age groups.
\end{abstract}

Key words: audiometry, epidemiology, hearing loss, NHANES, noise exposure, prevalence, rehabilitation, thresholds, tinnitus, veterans.

\section{INTRODUCTION}

Hearing loss and tinnitus are the two most prevalent service-connected disabilities for U.S. veterans, including those who served in Operation Iraqi Freedom or Operation Enduring Freedom [1]. Currently, in the Department of Veterans Affairs (VA), more than 570,000 veterans are service-connected for hearing loss and more than 639,000 are service-connected for tinnitus, which means they qualify for monthly compensation and/or VA clinical services related to these auditory disorders [1]. Nearly 476,000 hearing aids were issued by VA audiology clinics in fiscal year 2009. The cost for hearing aids, accessories, assistive devices, and associated clinical services exceeded \$393 million that year alone [2]. The number of

\footnotetext{
Abbreviations: $\mathrm{HL}=$ hearing level, $\mathrm{MEC}=$ mobile examination center, NCHS = National Center for Health Statistics, NHANES $=$ National Health and Nutrition Examination Survey, NHIS = National Health Interview Survey, PTA = pure tone average, VA = Department of Veterans Affairs, VAMC = VA medical center, VBA = Veterans Benefits Administration, VHA = Veterans Health Administration.

*Address all correspondence to Robert L. Folmer, PhD; National Center for Rehabilitative Auditory Research, Portland VA Medical Center, 3710 SW U.S. Veterans Hospital Road, Portland, OR 97239; 503-220-8262, ext 51868; fax: 503-721-1402. Email: Robert.Folmer@va.gov DOI:10.1682/JRRD.2010.07.0138
} 
veterans receiving VA compensation and services for hearing loss and tinnitus continues to increase annually. Consequently, auditory dysfunction in veterans and U.S. military personnel has become a priority for VA and Department of Defense research, rehabilitation, and prevention efforts.

The statistics just stated reflect numbers of veterans who received hearing healthcare services from VA medical centers (VAMCs) or compensation payments from the Veterans Benefits Administration (VBA). However, the majority of veterans in the United States do not use VAMCs or VA clinics for healthcare and do not receive VBA compensation [3]. Therefore, the prevalence of hearing loss and tinnitus among U.S. veterans is unknown. This study used National Health and Nutrition Examination Survey (NHANES) data to estimate the prevalence of these auditory conditions among male veterans.

NHANES is a program of studies that assess the health and nutritional status of adults and children in the United States. Designed and conducted by the National Center for Health Statistics (NCHS - a center within the Centers for Disease Control and Prevention), the survey combines interviews and physical examinations. According to the Web site of NCHS (http://www.cdc.gov/nchs /nhanes/about nhanes.htm), "the NHANES program began in the early 1960s and has been conducted as a series of surveys focusing on different population groups or health topics. In 1999, the survey became a continuous program that has a changing focus on a variety of health and nutrition measurements." The survey examines a sample that nationally represents 5,000 or more people each year. These participants are selected from counties across the country, with 15 counties visited by NHANES each year. The survey sample is selected to represent the U.S. population of all ages. To produce reliable statistics, NHANES oversamples persons 60 and older, African Americans, and Hispanics. NHANES data collected through 2006 are posted online and include demographic information; pure tone audiometric thresholds for subsets of participants; and questions about military service, noise exposure, and tinnitus perception.

Because many veterans were exposed to loud sounds during military service, we anticipated that they would exhibit higher (that is, poorer) pure tone thresholds than age-matched groups of nonveterans. Furthermore, we predicted that males with histories of loud noise exposure would exhibit higher pure tone thresholds than agematched males who reported less noise exposure. Finally, we hypothesized that the chronic tinnitus prevalence would be significantly greater among male veterans than the prevalence among male nonveterans and that tinnitus prevalence among males with histories of loud noise exposure would be greater than that among age-matched males with less noise exposure. Tinnitus is the perception of ringing, buzzing, hissing, or other noises in the ears or head in the absence of external sources for these sounds. These perceptions can be transient, intermittent, occasional, or constant. "Chronic" tinnitus is present all or most of the time during a person's waking hours. Like sensorineural hearing loss, chronic tinnitus more likely occurs in middle-aged and older people, especially those who have been repeatedly exposed to loud sounds without using hearing protection devices.

\section{METHODS}

The following description of the NHANES was taken, in part, from its Web site (http://www.cdc.gov/nchs /nhanes/about nhanes.htm) (verbatim passages are contained within quotation marks): "The NHANES interview includes demographic, socioeconomic, dietary, and health-related questions. The examination component consists of medical, dental, and physiological measurements, as well as laboratory tests administered by trained medical personnel. ... . Health interviews are conducted in respondents' homes. Health measurements are performed in specially-designed and equipped mobile [examination] centers [MECs], which travel to locations throughout the country. The study team consists of a physician, medical and health technicians, as well as dietary and health interviewers. . . . All participants visit the physician. Dietary interviews and body measurements are included for everyone. All but the very young have a blood sample taken and will have a dental screening. Depending upon the age of the participant, the rest of the examination includes tests and procedures to assess various aspects of health . . . . In general, the older the individual [is], the more extensive the examination." NHANES strives to facilitate and encourage participation. "Transportation is provided to and from the mobile center if necessary. Participants receive compensation and a report of medical findings .... A All information collected in the survey is kept strictly confidential. [Participant] privacy is protected by public laws." Deidentified data are available for analysis on the NHANES Web site. 


\section{NHANES Audiometry Examination}

Audiological data collected in NHANES are divided into questionnaire and examination components. The questionnaire includes questions about tinnitus and noise exposure and is completed by all subjects. During the 1999 to 2004 survey cycles, additional noise exposure data were recorded in the Occupational History Questionnaire. These questions were revised in the 2005 to 2006 NHANES and were included in the audiometry questionnaire. The NHANES Audiometry Examination Component consists of four parts and was administered to a subsample of participants in the MEC. The following description of the Audiometry Examination was taken from its online documentation (http://www.cdc.gov/nchs /data/nhanes/nhanes 05 06/aux d.pdf)—verbatim passages are contained within quotation marks:

1. "A pre-exam audiometric questionnaire: This is a series of questions to identify conditions that would affect how audiometric testing is conducted, or how results are interpreted. Questions include whether the subject has ear tubes, a current cold or ear problem, or recent loud noise exposure. ...

2. A brief otoscopic screening (physical) exam[ination] of the ear canals and eardrums: This was performed to identify abnormalities which would require alternate audiometric procedures or influence interpretation of test results, and to identify conditions which might require medical referral. The exam[ination] screened for excessive or impacted ear cerumen (wax), physical abnormalities, or collapsing external ear canals.

3. Tympanometry: This is an objective assessment of middle ear function by testing the mobility of the eardrum in response to changes in air pressure within the ear canal. It was used to identify middle ear pathologies that might contribute to hearing loss....

4. Pure tone air conduction audiometry: This measures hearing sensitivity by presenting pure tone signals to the ear through earphones and by varying the intensity of the signals until a subject's hearing threshold at that frequency is determined. Testing was performed at [seven] frequencies ..." from 500 to 8,000 Hz.

"Instrumentation for the Audiometry Component included an Interacoustics Model AD226 [Assens, Denmark] audiometer with standard TDH-39 headphones [Farmingdale, New York] and Etymotic EarTone 3A insert earphones [Elk Grove Village, Illinois]. Tympanometry was performed using a Micro Audiometrics Earscan Acoustic Impedance Tympanometer [Murphy, North
Carolina].” Between 1999 and 2004, “all Audiometry Component sections were performed by a trained examiner on examinees" aged 20 to 69 years "in a dedicated, sound-isolating room in the mobile examination center (MEC).” In 2005 and 2006, the same procedures were conducted on participants aged 70 or older and not on individuals aged 20 to 69. During both periods, "hearing threshold testing was conducted on both ears of examinees at the same seven frequencies $(500,1,000,2,000$, $3,000,4,000,6,000$, and $8,000 \mathrm{~Hz}$ ). Testing was conducted according to a modified Hughson Westlake procedure using the automated testing mode of the audiometer, except as indicated below. The effective range for automated audiometric testing was from -10 to 100 decibels (dB) [HL (hearing level)] at 500 to $6,000 \mathrm{~Hz}$ and -10 to $90 \mathrm{~dB}$ at $8,000 \mathrm{~Hz}$. Thresholds could be tested through $120 \mathrm{~dB}(110 \mathrm{~dB}$ at 8,000 Hz) using manual audiometric mode. Observed values, therefore, varied between -10 and $120 \mathrm{~dB}$. If an examinee did not respond to the signal tone at any level for one or more frequencies because of deafness or severe hearing loss, a threshold level [code] of 666 was entered. Manual testing was also conducted when the examinee could not operate the response switch or responded too slowly for the audiometer to accurately record the response...."

"The audiometric test room was required to meet or exceed the specifications of ANSI [American National Standards Institute] S3.1-1991 for ears covered testing. A Quest Model BA-201-25 Bioacoustic Simulator and Octave Band Monitor [Quest Technologies; Oconomowoc, Wisconsin] was used to continuously measure the background noise levels in the audiometric test room during audiometric examinations. Pure tone audiometric testing was not performed if ambient noise levels in the test booth exceeded maximum permissible levels .... As an additional quality measure, all audiograms, whether conducted in automated or manual mode, tested the $1,000 \mathrm{~Hz}$ frequency twice in each ear as a measure of the reliability of the subject's responses. Pure tone audiograms were not accepted if there was more than a $10 \mathrm{~dB}$ difference between them. For further details regarding any of these procedures, analysts should consult the NHANES Audiometry/Tympanometry Procedures Manual” [4].

"In some instances, if a pure tone audiometric signal is sufficiently loud, it can 'cross over' and be heard by the opposite ear via bone conduction. When this occurs, it is difficult to determine if the threshold obtained is truly the threshold of the test ear, or an artifact of the nontest ear 
(which may have better hearing).” For NHANES 1999 to 2006, "a crossover retesting protocol was performed whenever the observed threshold at any given frequency was poorer [higher] in one ear than the other by $25 \mathrm{~dB}$ at 500 and $1,000 \mathrm{~Hz}$; or $40 \mathrm{~dB}$ at any higher frequency. Retesting was accomplished using insert earphones, which are smaller and have less direct contact with the head. Thus, a much louder stimulus is required before crossover occurs. Due to the complexity of the procedure, masking was not employed in this survey."

"On a continuous basis, a consulting Audiologist performed a clinical review of all data for each subject as it was received, checking for quality and consistency. In addition, a computerized data editing program was developed to check for logical inconsistencies in the data and technician errors, and to cross-check other issues affecting data quality (consistency in identifying potential instances where crossover effects might have occurred, assurance of randomization of the initial test ear, etc.). [Back-end] Edits of the data were performed as needed when errors were detected."

Subjects were selected for NHANES according to a complex survey-design algorithm for the questionnaire and examination components. Briefly stated, the design involves randomly selecting counties, neighborhoods, households, and household members. Complex survey designs such as that used in NHANES result in each participant assigned strata, clusters, and survey weights that must be accounted for during data analysis. A subject's survey weight is interpreted as the number of people in the general U.S. population represented by that subject. Weights are derived from numerous variables and estimation procedures that are described in the NHANES documentation. Our analysis of the 1999 to 2004 data uses a 6-year weighting scheme, while the 2005 to 2006 data are analyzed separately using 2-year survey weights. For more information on the use of sample weights in NHANES data analysis, refer to the current NHANES Analytic and Reporting Guidelines [5].

This study analyzed the following NHANES data (extracted from the NCHS Web site http://www.cdc.gov /nchs/nhanes/about nhanes.htm) collected from male participants between 1999 and 2006. Females were excluded from these analyses because the numbers of female veterans in each age category were too small to allow meaningful statistical comparisons with nonveteran females in the samples.
Each participant's age and sex were determined at the time of the interview. Veteran status was defined as an affirmative response to the following question: "Did you ever serve in the Armed Forces of the United States?" For years 1999 to 2004, we defined noise exposure as a composite of occupational and nonoccupational noise. Nonoccupational noise exposure was defined as unprotected exposure to firearms, loud music, or power tools for an average of at least once a month for at least 1 year. Occupational noise exposure was defined as current exposure to loud noise while at work. NHANES questionnaires do not differentiate noise exposure during military service from other types of occupational noise exposure, so current noise exposure data were used for our analysis. This methodology is appropriate, since NHANES sampling does not include Active Duty military personnel. For NHANES 1999 to 2004, participants were identified as "noise-exposed" if they answered "Yes" to the question "Outside of work, have you ever been exposed to firearms noise for an average of at least once a month for a year?" and if they answered "No" to the question "Have you ever worn hearing protection devices when exposed to firearms noise?” or if they answered "Yes" to the question "Outside of work, have you ever been exposed to other types of loud noise, such as noise from power tools or loud music, for an average of at least once a month for a year? [By loud noise I mean noise so loud that you had to speak in a raised voice to be heard.]" and if they answered "No" to the question "Have you ever worn hearing protection devices when exposed to these loud noises?" or if they answered "Yes" to "At your job as a(n) \{OCCUPATION\} for \{EMPLOYER\}, are you currently exposed to loud noise? [By loud noise, I mean noise so loud that you have to speak in a raised voice to be heard.]."

Noise exposure questions changed for the 2005 to 2006 NHANES cycle. For these years, we identified a subject as noise-exposed if he identified any history of occupational or nonoccupational noise exposure for 5 or more hours a week, during which he "Rarely/Seldom" or "Never" used hearing protection. For NHANES 2005 to 2006, a significant history of noise exposure was assumed if participants answered "Yes" to the question "Have you ever had a job where you were exposed to loud noise for 5 or more hours a week? [By loud noise I mean noise so loud that you had to speak in a raised voice to be heard.]" or answered "Yes" to the question "Outside of a job, have you ever been exposed to steady loud 
noise or music for 5 or more hours a week? [This is noise so loud that you have to raise your voice to be heard. Examples are noise from power tools, lawn mowers, farm machinery, cars, trucks, motorcycles, or loud music.]" and if participants answered "Rarely/Seldom" or "Never" in response to, "How often do you wear hearing protection devices (ear plugs, ear muffs) when exposed to loud sounds or noise? (Include both job and off work exposures.).”

Outcomes for this study included pure tone air conduction audiometric thresholds (NHANES 1999-2006) and prevalence of tinnitus (NHANES 1999-2004). Participants were said to experience chronic tinnitus if they answered "Yes" to the question "In the past 12 months, have you ever had ringing, roaring, or buzzing in your ears?" and if they answered "Almost always" to the follow-up question "How often did this happen? Would you say . . . ?" (The other response choices were "At least once a day," "At least once a week," "At least once a month," or "Less frequently than once a month.") We did not analyze prevalence of tinnitus data for NHANES 2005 to 2006 because the wording and structure of tinnitus-related questions changed significantly for the survey during this time compared with questions during 1999 to 2004. Therefore, combining or comparing tinnitus prevalence data for these different NHANES questionnaires is not feasible.

\section{Data Analysis}

NHANES data management, analysis, and reporting were conducted using SAS software, version 9.2 (SAS Institute Inc; Cary, North Carolina). Statistical comparisons were made between veteran and nonveteran male participants and between groups of participants who did or did not report a significant history of noise exposure.

We estimated the chronic tinnitus prevalence and mean pure tone thresholds at each audiometric test frequency using the proper survey weights. We based the standard error of each estimate on a Taylor's series linearization using the complex design features [6]. Tests of the null hypothesis of no difference in chronic tinnitus prevalence between veterans and nonveterans are based on the Rao-Scott Likelihood Ratio chi-square test. Similar tests for pure tone thresholds at each audiometric test frequency are based on the Wald $F$ statistic.

Our primary objective of this study was to evaluate differences in audiometric configurations and the prevalence of tinnitus between the U.S. male veteran and non- veteran populations, while controlling for age and noise exposure. Many respondents to NHANES did not satisfy the eligibility requirements of our study for one or more of the following reasons: they were younger than 20 years of age, were women, or were missing responses to one or more variables needed to conduct the analysis. In standard statistical analysis, ineligible subjects are ignored and attention is focused on those respondents within the eligible ranges. This procedure is not possible when complex survey data are analyzed because survey weights were defined for the entire sample of NHANES respondents. Simply excluding the ineligible subjects and computing separate statistics within each age/veteran status subgroup would cause the standard errors to be estimated incorrectly. Accordingly, we used domain analysis [6] to compute proper subgroup statistics for both eligible and ineligible subjects in the sample. Results are reported exclusively for the subgroups of interest in this study.

\section{RESULTS}

\section{Pure Tone Audiometric Thresholds}

From 1999 to 2004, NHANES collected usable audiometric data from 5,742 individuals aged 20 through 69 years. Of these, 2,545 males provided data suitable for analysis. Most of the remaining participants were women $(n=3,068)$, and some $(n=129)$ of the remaining men had invalid data related to survey weights, veteran status, noise exposure, or audiometric results. The 2005 to 2006 NHANES yielded data on 10,348 participants, of whom 386 were men aged 70 and older with valid audiometric data. The majority of exclusions for 2005 to 2006 were participants under 70 years old $(n=9,466)$, of which the remainder were women $(n=431)$ or individuals with invalid data $(n=65)$.

We analyzed audiometric data collected from 2,931 males who participated in NHANES between 1999 and 2006. Table 1 lists the number of participants in each age category and the numbers of male veterans and nonveterans in each age category. Note that the percentage of veterans in each age category increases with the age of participants. Some subjects did not have measurable thresholds for certain frequencies, so sample sizes for certain frequencies might be slightly lower than those sizes shown in Table 1.

For this analysis, pure tone thresholds obtained at each frequency tested for the left and right ears were 
averaged for each male participant. Figure 1 shows average pure tone thresholds for each test frequency and age group. As expected, thresholds for each test frequency

Table 1.

Numbers of male participants in each category who provided pure tone audiometric data for analysis (National Health and Nutrition Examination Survey, 1999-2006).

\begin{tabular}{cccc}
\hline Age (yr) & Veterans (\%) & Nonveterans (\%) & Total \\
\hline $20-29$ & $34(6.04)$ & $529(93.96)$ & 563 \\
$30-39$ & $74(14.31)$ & $443(85.69)$ & 517 \\
$40-49$ & $84(16.12)$ & $437(83.88)$ & 521 \\
$50-59$ & $158(36.16)$ & $279(63.84)$ & 437 \\
$60-69$ & $231(45.56)$ & $276(54.44)$ & 507 \\
$70-79$ & $155(65.96)$ & $80(34.04)$ & 235 \\
$80-89$ & $109(72.19)$ & $42(27.81)$ & 151 \\
\hline Total & $845(28.83)$ & $2,086(71.17)$ & 2,931 \\
\hline \hline
\end{tabular}

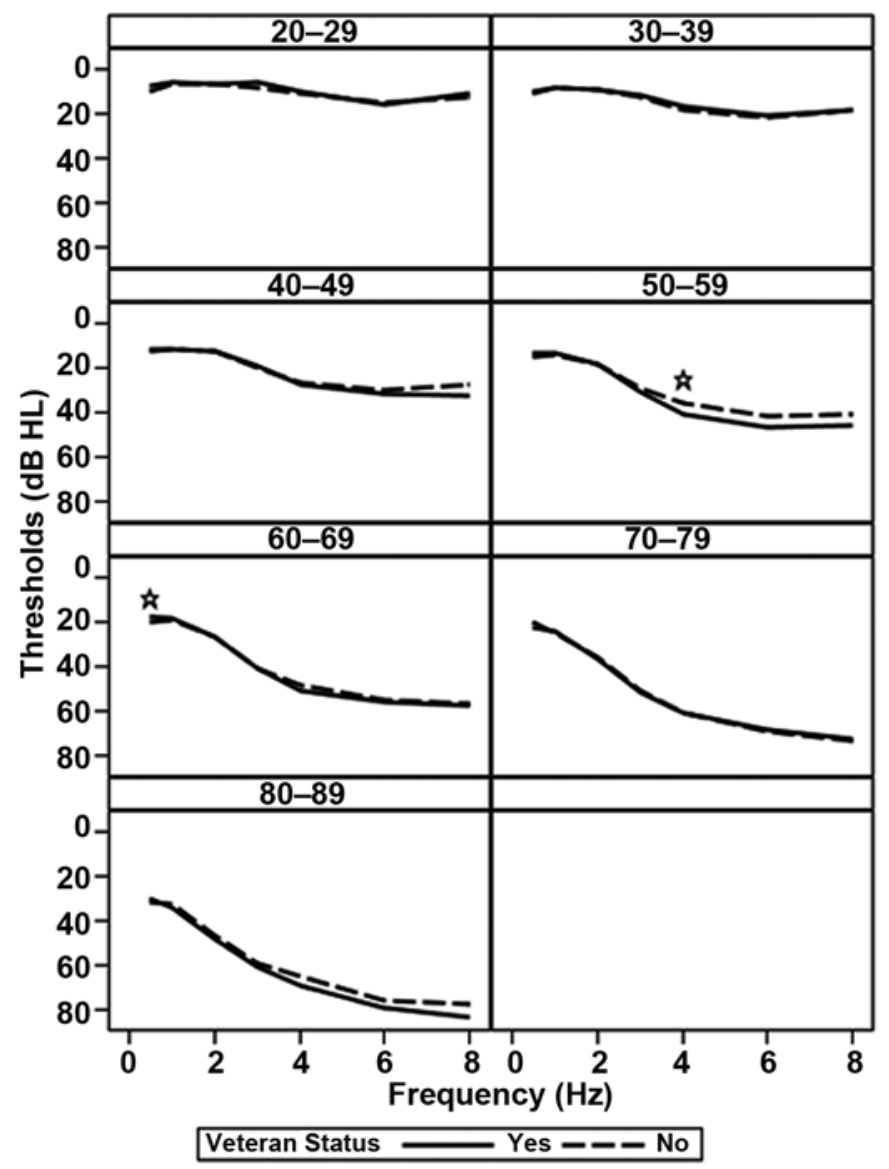

Figure 1.

Audiograms for National Health and Nutrition Examination Survey respondents (1999-2006) by age category. Statistically significant contrasts are indicated by stars. tended to be higher (that is, poorer) in successively older age groups of both veterans and nonveterans. In general, most audiometric thresholds for veterans are not significantly different from those for nonveterans, with the following exceptions: $500 \mathrm{~Hz}$ for 60- to 69-year-olds (higher threshold for nonveterans, $p=0.04$ ); $4,000 \mathrm{~Hz}$ for 50 - to 59-year-olds (higher threshold for veterans, $p=$ $0.04)$. In addition, 50- to 59-year-old veterans exhibited higher thresholds for $6,000 \mathrm{~Hz}(p=0.06)$ and $8,000 \mathrm{~Hz}$ tones $(p=0.07)$ than those for nonveterans, but these differences did not quite achieve the statistical significance level of $p<0.05$.

Figure 2 shows average pure tone thresholds for each test frequency and age group for veterans and nonveterans who reported a history of significant noise exposure. The majority of thresholds for each test frequency are similar for participants within the same age group. However, one

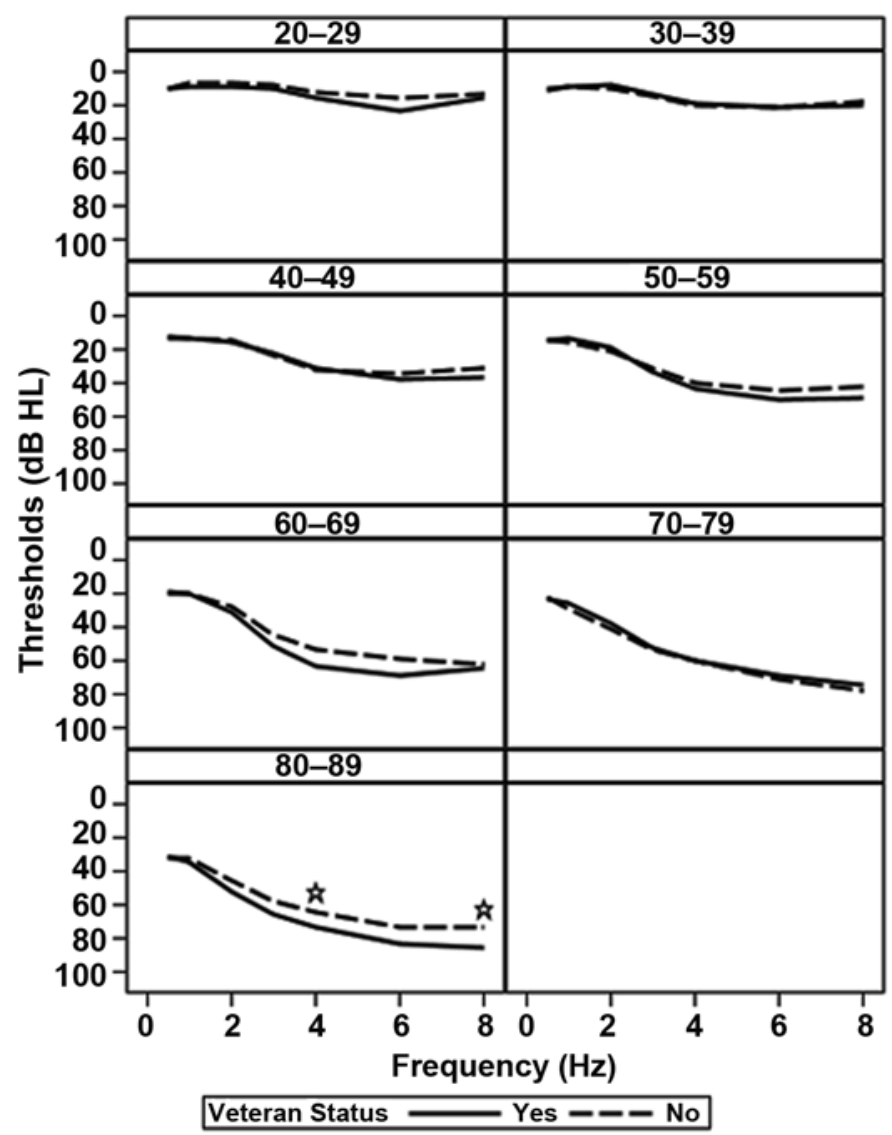

Figure 2.

Audiograms for noise-exposed National Health and Nutrition Examination Survey respondents (1999-2006) by age category. Statistically significant contrasts are indicated by stars. 
set of statistical differences is apparent in Figure 2: veterans aged 80 years or older with histories of noise exposure exhibited higher (poorer) thresholds for 4,000, 6,000, and $8,000 \mathrm{~Hz}$ tones than those thresholds of nonveterans in the same age group who also reported higher levels of noise exposure. In addition, 80+-year-old veterans with noise exposure histories exhibited higher thresholds than those of nonveterans with noise exposure for $3,000 \mathrm{~Hz}(p=$ $0.07)$, but the difference did not achieve the statistical significance level of $p<0.05$.

Figure 3 shows average pure tone thresholds for each test frequency and age group for veterans and nonveterans who did not report histories of significant noise exposure. Again, most thresholds for each test frequency are similar for participants within the same age group. Exceptions include higher thresholds at most test frequencies for nonveterans in the 20 to 29 age category with low-noise exposure than those for veterans in the same age range with histories of low-noise exposure. Only 14 veterans were in this subgroup, and this small sample of veterans had exceptionally good hearing at all frequencies tested. Furthermore, pure tone thresholds among the nonveterans were skewed by a handful of respondents with high thresholds for mid- or lower frequencies (e.g., $>40 \mathrm{~dB} \mathrm{HL}$ at $1 \mathrm{kHz}$ ). Since thresholds are likely to be correlated across frequencies, it is not surprising that statistically significant results are observed over the range of test frequencies in this age group. Pure tone thresholds for nonveterans in the 20 to 29 age category with low noise exposure $(n=300)$ are statistically similar to thresholds obtained from 20- to 29-year-old nonveterans who reported higher levels of noise exposure $(n=224)$.

\section{Prevalence of Tinnitus}

From 1999 to 2004, NHANES collected questionnaire data from 31,126 participants, 7,169 of whom were males 20 years and older with valid data. Most excluded subjects were under the age of $20(n=15,794)$; of the remainder, a large number were women $(n=8,109)$ and a small number $(n=54)$ had invalid data for tinnitus, veteran status, or noise exposure.

Table 2 contains tinnitus prevalence data for 7,169 male participants (20 years and older) in NHANES from 1999 to 2004, 2,174 (30.3\%) of whom were veterans. The overall chronic tinnitus prevalence for all males in this sample is 7.1 percent. The prevalence of tinnitus for veterans is 11.7 percent; for nonveterans it is 5.4 percent. This difference between veterans and nonveterans is sta-

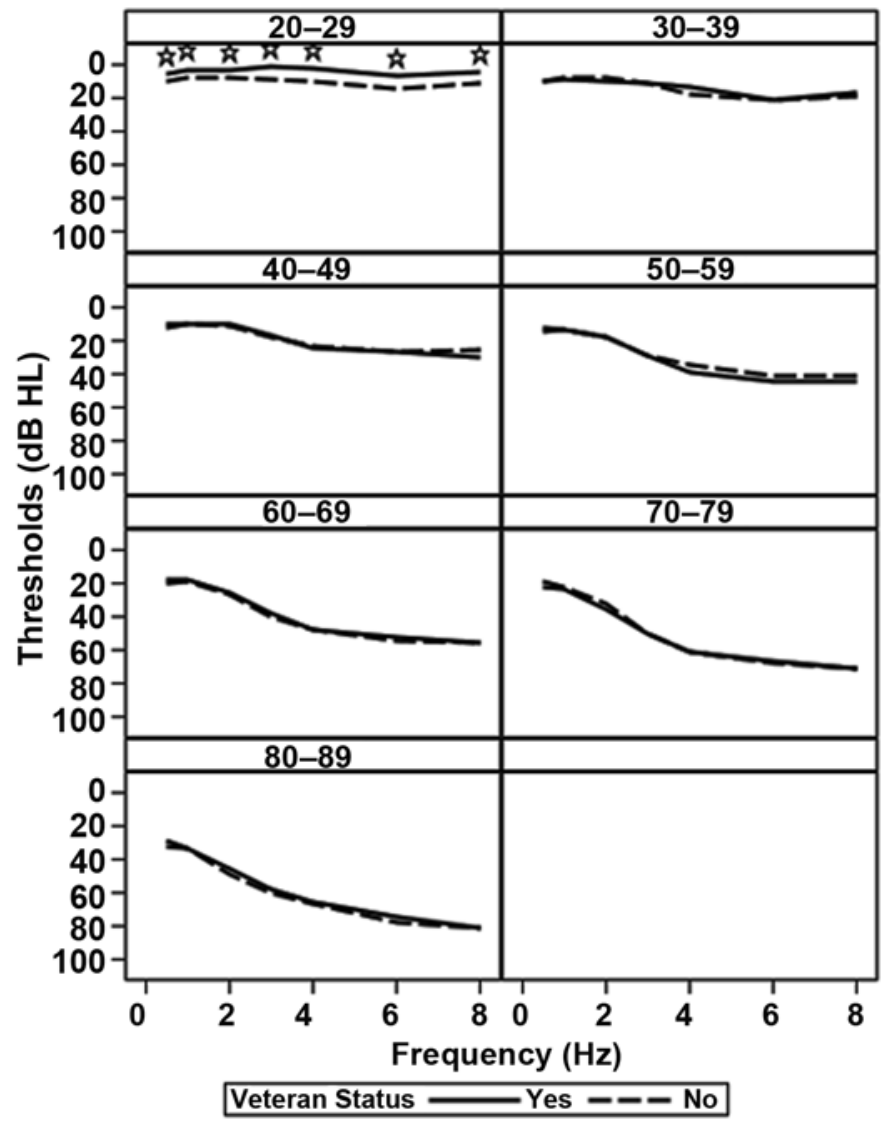

Figure 3.

Audiograms for National Health and Nutrition Examination Survey respondents (1999-2006) reporting no significant noise exposure by age category. Statistically significant contrasts are indicated by stars.

tistically significant (Chi-square statistic $=58.9 ; p<$ 0.001 ). The odds ratio for the association between veteran status and tinnitus is 2.3 (95\% confidence interval $=$ 1.9 to 2.9 ), indicating more than a twofold higher prevalence of tinnitus among veterans than nonveterans. In general, the prevalence of tinnitus increases from the 20 to 29 through the 60- to 69-year-old age categories, then decreases for the 70- to 79- and 80+-year-old categories. Compared with nonveterans, veterans have statistically greater prevalence of tinnitus in the 50 to $59(p=0.04)$ and 60 to $69(p<0.01)$ age categories.

Table 3 contains tinnitus prevalence data for male veterans and nonveterans who participated in NHANES between 1999 and 2004 and who did or did not report a history of significant noise exposure, according to our definition. The chronic tinnitus prevalence among males who reported significant noise exposure is 8.8 percent; 
Table 2.

Prevalence of tinnitus among males, from National Health and Nutrition Examination Survey, 1999-2004.

\begin{tabular}{|c|c|c|c|c|c|c|c|c|c|c|}
\hline \multirow{2}{*}{$\begin{array}{c}\text { Age } \\
\text { Category } \\
(y r)\end{array}$} & \multicolumn{3}{|c|}{ Veterans } & \multicolumn{3}{|c|}{ Nonveterans } & \multicolumn{3}{|c|}{ Total } & \multirow{2}{*}{$\begin{array}{c}p \text {-Value } \\
\text { Veterans vs } \\
\text { Nonveterans }\end{array}$} \\
\hline & $n$ & $\%$ & SE (\%) & $n$ & $\%$ & SE (\%) & $n$ & $\%$ & SE (\%) & \\
\hline $20-29$ & 63 & 0.9 & 0.9 & 1,138 & 1.5 & 0.4 & 1,201 & 1.5 & 0.4 & 0.56 \\
\hline 30-39 & 148 & 5.2 & 1.9 & 994 & 3.1 & 0.6 & 1,142 & 3.4 & 0.6 & 0.23 \\
\hline $40-49$ & 208 & 5.0 & 2.0 & 1,014 & 6.4 & 0.9 & 1,222 & 6.1 & 0.9 & 0.55 \\
\hline $50-59$ & 341 & 15.2 & 2.2 & 591 & 9.8 & 2.0 & 932 & 12.1 & 1.6 & 0.04 \\
\hline $80+$ & 380 & 10.1 & 1.8 & 242 & 8.4 & 1.7 & 622 & 9.5 & 1.2 & 0.51 \\
\hline Total & 2,174 & 11.7 & 0.8 & 4,995 & 5.4 & 0.5 & 7,169 & 7.1 & 0.5 & $<0.001$ \\
\hline
\end{tabular}

Table 3.

Prevalence of tinnitus by noise exposure among males, from National Health and Nutrition Examination Survey, 1999-2004.

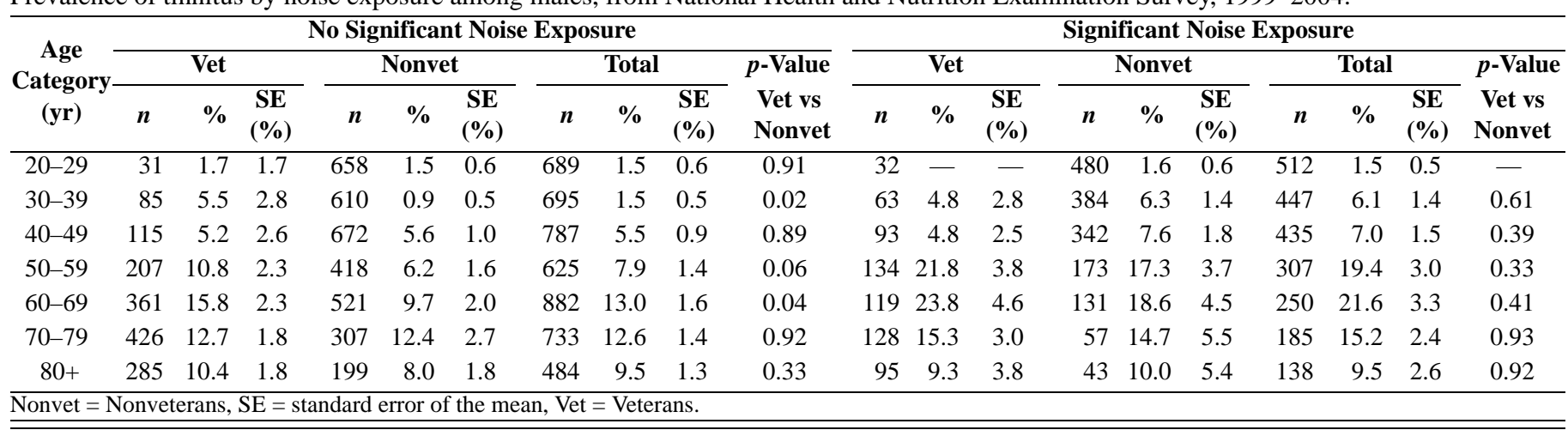

the prevalence among males who reported less noise exposure is 6.1 percent. This difference is statistically significant (chi-square statistic $=13.7 ; p<0.001$ ). Among males who had a history of noise exposure, no statistically significant differences were found in tinnitus prevalence between veterans and nonveterans for any of the age categories. However, for males who reported no significant noise exposure, veterans had higher prevalence of tinnitus than nonveterans in the 30 to 39 ( $p=$ $0.02)$ and 60 to $69(p<0.04)$ age groups; the disparity between 50 - to 59-year-olds in these subgroups approached statistical significance $(p=0.06)$.

\section{DISCUSSION}

To our knowledge, this study is the first that uses NHANES data to provide information about audiometric thresholds and prevalence of tinnitus among a sample of all male veterans in the United States, not just those who have been evaluated at VAMCs or VA clinics. Because many veterans were exposed to loud sounds during military service, we anticipated that compared with agematched groups of nonveterans, veterans would exhibit higher pure tone thresholds. Furthermore, before we began our analyses of NHANES data, we predicted that males with histories of loud noise exposure would exhibit higher pure tone thresholds than those of age-matched males who reported less noise exposure. In general, neither of these predictions was supported by NHANES data, with the exception of higher frequency thresholds in noise-exposed veterans +80 years old.

Wilson et al. analyzed audiometric data collected from male veterans and nonveterans (aged 48-92 years) who participated in the Epidemiology of Hearing Loss Study between 1993 and 1995 [7]. The authors reported that pure tone thresholds, word recognition in quiet, and word recognition in competing message increased with age but were not significantly different for veterans and nonveterans. Also, no significant differences were found 
between participant groups on the Hearing Handicap Inventory for the Elderly questionnaire.

Why were pure tone thresholds of veterans and nonveterans similar in both of these studies? We propose the following explanations:

1. For averaged audiometric data, age is the most important criterion for determining pure tone thresholds at each test frequency [8-10]. In this study, the prevalence and effects of presbycusis appear to be similar and evenly distributed among male veterans and nonveterans.

2. Although veterans are sometimes exposed to loud sounds during military service, many veterans and nonveterans are also exposed to loud sounds during recreational and nonmilitary occupational activities. Occupational or recreational noise exposure can occur for decades during a person's life, a much longer time than most veterans spend in the military. In addition to the aging process, this nonmilitary noise exposure could also contribute to similarities in audiometric patterns exhibited by veterans and nonveterans.

One pattern of audiometric differences did occur in this analysis of NHANES data: compared with nonveterans 80 or older with histories of significant noise exposure, veterans in the same age group also with histories of significant noise exposure exhibited higher thresholds for $3,4,6$, and $8 \mathrm{kHz}$ tones. The relatively small number of nonveterans in the latter category (21) may possibly be skewing the results. However, if this trend holds in a larger sample of veterans aged $80+$ years, it might provide evidence for the theory that noise exposure early in life increases the chance of hearing loss later in life [11].

Previous studies of group audiometric data reported one of the trends observed in this article: pure tone thresholds increase with the subjects' age [8-10]. Some studies attempted to estimate the prevalence of hearing loss in a population based on audiometric data [8-9,1213]. Such estimations require a definition of "hearing loss.” Using Ventry and Weinstein criteria (40 dB or greater thresholds for 1 and $2 \mathrm{kHz}$ ) [14], Reuben et al. reported a hearing loss prevalence of 14.2 percent in a sample of 2,506 people aged 55 to 74 years [12]. When Reuben et al. used high-frequency (1, 2, and $4 \mathrm{kHz}$ ) pure tone average (PTA) criteria, the prevalence of hearing loss in this population rose to 35.1 percent [12]. In Mocicki et al.'s study of 2,293 people aged 57 to 89 years, they defined hearing loss as "a threshold level greater than $20 \mathrm{~dB}$ above audiometric zero for at least one frequency from 0.5 to $4 \mathrm{kHz}$ " in the better ear [8]. Using this definition, Mocicki et al. reported a hearing loss prevalence of 83 percent for their study population. Cruickshanks et al. defined hearing loss as "a pure-tone average of thresholds at 500,1,000, 2,000, and $4,000 \mathrm{~Hz}$ greater than $25 \mathrm{~dB}$ ” in the worse ear [10]. In their study of 3,753 people aged 48 to 92 years, Cruickshanks et al. reported a hearing loss prevalence of 45.9 percent [10]. In Agrawal et al.'s analysis of NHANES data from 2003 to 2004 [13], they used similar criteria as Cruickshanks et al. [10] used to define "speech-frequency hearing loss." According to these criteria, Agrawal et al. estimated that 16.1 percent of all people in the United States aged 20 to 69 years experience speech-frequency hearing loss [13].

Since this study focuses on veterans, we analyzed NHANES audiometric data according to criteria outlined in the VA definition of disability due to impaired hearing: "Impaired hearing will be considered to be a disability when the auditory threshold in any of the frequencies 500 , $1,000,2,000,3,000,4,000$ Hertz is 40 decibels or greater; or when the auditory thresholds for at least three of the frequencies $500,1,000,2,000,3,000$, or 4,000 Hertz are 26 decibels or greater; or when speech recognition scores using the Maryland CNC [Computer Numerical Control] Test are less than 94 percent” [15]. Table 4 contains the percentages of male veterans in each age category who meet or exceed these VA audiometric criteria for disability due to hearing loss. As expected, these percentages increase with age, beginning with the 40- to 49- year-old category. Because NHANES data do not include speech recognition scores, the numbers in Table 4 probably represent underestimations of the percentages of veterans who meet VA audiometric criteria for disability due to hearing loss.

Table 4.

Percentage \pm SE (\%) of male veterans who meet or exceed Department of Veterans Affairs audiometric criteria for disability due to hearing loss.

\begin{tabular}{cc}
\hline Age Category (yr) & Percentage \pm SE (\%) \\
\hline $20-29$ & $16.2 \pm 9.0$ \\
$30-39$ & $14.2 \pm 4.0$ \\
$40-49$ & $33.9 \pm 4.8$ \\
$50-59$ & $64.8 \pm 3.9$ \\
$60-69$ & $81.9 \pm 3.1$ \\
$70-79$ & $93.8 \pm 2.4$ \\
$80+$ & $98.8 \pm 1.2$ \\
\hline
\end{tabular}

$\overline{\mathrm{SE}}=$ standard error of the mean. 
The overall prevalence of tinnitus for adult males in this study $(7.1 \%)$ is slightly lower than the tinnitus prevalence (8.4\%) reported by Ries [16] for all adults who participated in the U.S. National Health Interview Survey (NHIS) Hearing Supplement in 1990. For adults 50 years or older, Ries reported a tinnitus prevalence of 12.1 percent [16]. In Adams et al.'s analysis of NHIS data collected in 1996, they reported a tinnitus prevalence of 11.7 percent for males 65 years or older [17]. In the present study, the tinnitus prevalence for males 60 years or older is 13.6 percent. Ries, Adams et al., Brown, Hoffman and Reed, and other authors reported that tinnitus prevalence increases with increasing age of subjects in a sample [16-19]. Data from the present study agree with this trend. Hoffman and Reed also demonstrated that the prevalence of tinnitus is positively correlated with PTA audiometric thresholds for adults aged 25 years or older [19]. Because PTAs usually increase as people age (Table 5), increases in hearing loss are the primary contributors to increases in tinnitus prevalence that occur from younger to older age groups.

Before we began our analyses of NHANES data, we predicted that the chronic tinnitus prevalence would be significantly greater among male veterans than the prevalence among male nonveterans. Data in the 1999 to 2004 NHANES samples support this hypothesis. In their analysis of 1994 NHIS data, Adams and Marano also reported a higher prevalence of tinnitus for veterans than the prevalence for nonveterans [20]. Ries reported that a significantly higher percentage of veterans experienced "bothersome tinnitus" compared with the percentage of a group of nonveterans who participated in the 1990 NHIS Hearing Supplement [16]. Most likely, veterans' exposure to loud sounds (e.g., weapons fire, aircraft, explo-

\section{Table 5.}

Pure tone average thresholds (in decibel hearing level for 0.5, 1.0, 2.0, and $4.0 \mathrm{kHz}) \pm$ standard error of the mean for males in each category (National Health and Nutrition Examination Survey, 1999-2006).

\begin{tabular}{crrc}
\hline $\begin{array}{c}\text { Age Category } \\
(\mathbf{y r})\end{array}$ & Veterans & Nonveterans & $\boldsymbol{p}$-Value \\
\hline $20-29$ & $7.8 \pm 2.0$ & $8.8 \pm 0.4$ & 0.59 \\
$30-39$ & $11.2 \pm 0.9$ & $11.7 \pm 0.5$ & 0.60 \\
$40-49$ & $15.7 \pm 1.0$ & $15.9 \pm 0.7$ & 0.88 \\
$50-59$ & $21.7 \pm 0.9$ & $21.0 \pm 1.1$ & 0.59 \\
$60-69$ & $28.5 \pm 1.1$ & $28.6 \pm 1.1$ & 0.90 \\
$70-79$ & $36.0 \pm 1.4$ & $35.0 \pm 1.8$ & 0.74 \\
$80+$ & $45.4 \pm 1.4$ & $44.3 \pm 2.0$ & 0.62 \\
\hline \hline
\end{tabular}

sions, or vehicles) during military service, combined with other factors (such as occupational and recreational noise exposure, the aging process, and coexisting medical conditions), contributed to the higher prevalence of tinnitus in this population.

Because tinnitus prevalence in this study is higher for veterans and for males with greater levels of noise exposure, efforts to prevent hearing loss need to be increased among military personnel, veterans, and other members of our society [21-22]. Providing and disseminating education programs to prevent hearing loss nationally should help increase hearing-protection strategies during occupational and recreational activities [23-25]. Consistent implementation of hearing protective behaviors by large numbers of individuals in the United States would help reduce the prevalence and severity of hearing loss and tinnitus over time [26-27].

\section{CONCLUSIONS}

Analysis of NHANES data showed that the overall chronic tinnitus prevalence is greater for veterans (11.7\%) than the prevalence for nonveterans (5.4\%), with statistically significant differences in the 50 to 59 and 60 to 69 age groups. Also, the prevalence of tinnitus among males who reported a noise exposure history is significantly higher than the prevalence among males who reported less noise exposure. However, with few exceptions, the pure tone hearing thresholds for veterans did not differ significantly from nonveteran audiograms; males who reported more noise exposure did not have substantially worse hearing than males the same age with less noise exposure. These surprising audiometric results probably occurred because the larger effect of age in our decade-by-decade comparisons obscured the small differences in pure tone thresholds, if they exist between groups (veterans vs nonveterans or noise-exposed vs non-noise-exposed males).

In the near future, hearing loss and tinnitus will likely remain the most prevalent service-connected disabilities among all U.S. veterans. In addition, increasing numbers of veterans will probably seek and receive VA compensation and medical and rehabilitative services for these conditions. As they plan for future costs of healthcare and compensation, the Veterans Health Administration (VHA) and the VBA should be able to use results of this study and its estimates of audiometric thresholds and tinnitus prevalence 
among male veterans in the United States. Clinicians, researchers, administrators, and policy makers in related fields may also be interested in these findings.

Additional studies should be undertaken to-

1. Include data from female veterans.

2. Investigate specific characteristics of veterans and nonveterans that contribute to the prevalence of hearing loss and tinnitus reported here.

3. Acquire more detailed information about the types and severity of hearing loss and tinnitus experienced by veterans and nonveterans.

4. Acquire information related to treatments and rehabilitative services participants received for auditory complaints/disorders.

Data from these proposed investigations would provide a more comprehensive understanding of auditory dysfunction experienced by veterans and nonveterans in the United States. Information of this kind should also facilitate the development, implementation, and evaluation of rehabilitative interventions and policies related to hearing disorders.

Although the number of veterans enrolled in the VA healthcare system has increased during the last few years, only 30 percent of veterans in the United States receive healthcare services at VAMCs and VA clinics [3]. VA medical budgets increased in real (inflation-adjusted) terms from \$16 billion in 1990 to nearly \$28 billion in 2004, even though the number of veterans in the United States decreased by 1 to 2 percent a year during that period. According to an analysis by Grotto and Jones [28], "a dramatic spike in disability claims during the last seven years has overwhelmed the VA and nearly doubled the cost of compensating wounded veterans. The bulk of the increases didn't come from veterans of the current wars in Iraq and Afghanistan, but from those who served years or even decades before. Veterans from the Vietnam and Persian Gulf eras accounted for roughly 84 percent of the rise in spending, which hit $\$ 34.3$ billion last year. The surge from past eras comes even as more soldiers than expected are returning home from Iraq and Afghanistan in need of care. With hundreds of thousands of troops still deployed, the VA already provides disability payments to nearly 200,000 veterans from the current conflicts, a number that is expected to balloon during the next 30 years" [28].

The VHA and VBA should prepare for an everincreasing number of veterans seeking care and compensation for hearing loss and tinnitus. Adding veterans of the current conflicts to the aging population of existing veterans ensures that auditory dysfunction will remain a top priority and expense for the VA.

\section{ACKNOWLEDGMENTS}

\section{Author Contributions:}

Study concept and design: R. L. Folmer, G. P. McMillan, D. F. Austin, J. A. Henry.

Acquisition of data: G. P. McMillan, R. L. Folmer.

Analysis and interpretation of data: R. L. Folmer, G. P. McMillan,

D. F. Austin, J. A. Henry.

Drafting of manuscript: R. L. Folmer, G. P. McMillan.

Critical revision of manuscript for important intellectual content:

R. L. Folmer, G. P. McMillan, D. F. Austin, J. A. Henry.

Statistical analysis: G. P. McMillan.

Study supervision: R. L. Folmer.

Financial Disclosures: The authors have declared that no competing interests exist.

Funding/Support: This material was based on work supported by a VA Rehabilitation Research \& Development Center of Excellence grant C4844C to the National Center for Rehabilitative Auditory Research (located at the Portland VAMC) in Portland, Oregon. Additional Contributions: The authors thank Dr. Diana Pope for inspiring and facilitating this project.

Participant Follow-Up: The authors do not plan to inform the participants of the publication of this study because of a lack of contact information.

\section{REFERENCES}

1. Annual Benefits report fiscal year 2009 [Internet]. Washington (DC): Veterans Benefits Administration; 2009. Available from: http://www.vba.va.gov/.

2. Beck LB. A 21st century approach to VA audiology care. Paper presented at: 2010 JDVAC. Proceedings of the Joint Defense Veterans Audiology Conference; 2010 Feb 22; Orlando, FL.

3. The potential cost of meeting demand for veterans' health care [Internet]. Washington (DC): Congressional Budget Office; 2005 [cited 2010 Jun 21]. Available from: http:// www.cbo.gov/doc.cfm?index $=6171 \&$ type $=0 \&$ sequence $=2 /$.

4. Audiometry/tympanometry procedures manual [Internet]. Atlanta (GA): National Center for Health Statistics; 2001. Available from: http://www.cdc.gov/nchs/data/nhanes/au.pdf/.

5. Analytic and reporting guidelines: National Health and Nutrition Examination Survey [Internet]. Hyattsville (MD): National Center for Health Statistics; 2006 [updated 2006 Sep]. Available from: http://www.cdc.gov/nchs/data/ nhanes/nhanes_03_04/ nhanes analytic guidelines dec 2005.pdf/. 
6. Lohr S. Sampling: Design and analysis. Pacific Grove (CA): Duxbury Press; 1999.

7. Wilson RH, Noe CM, Cruickshanks KJ, Wiley TL, Nondahl DM. Prevalence and degree of hearing loss among males in Beaver Dam cohort: Comparison of veterans and nonveterans. J Rehabil Res Dev. 2010;47(6):505-20.

[PMID: 20848364]

DOI:10.1682/JRRD.2009.10.0169

8. Mocicki EK, Elkins EF, Baum HM, McNamara PM. Hearing loss in the elderly: An epidemiologic study of the Framingham Heart Study Cohort. Ear Hear. 1985;6(4): 184-90. [PMID: 4043571] DOI:10.1097/00003446-198507000-00003

9. Gates GA, Cooper JC Jr, Kannel WB, Miller NJ. Hearing in the elderly: The Framingham cohort, 1983-1985. Part I. Basic audiometric test results. Ear Hear. 1990;11(4):247-56. [PMID: 2210098]

DOI:10.1097/00003446-199008000-00001

10. Cruickshanks KJ, Wiley TL, Tweed TS, Klein BE, Klein R, Mares-Perlman JA, Nondahl DM. Prevalence of hearing loss in older adults in Beaver Dam, Wisconsin. The Epidemiology of Hearing Loss Study. Am J Epidemiol. 1998; 148(9):879-86. [PMID: 9801018]

11. Kujawa SG, Liberman MC. Acceleration of age-related hearing loss by early noise exposure: Evidence of a misspent youth. J Neurosci. 2006;26(7):2115-23. [PMID: 16481444] DOI:10.1523/JNEUROSCI.4985-05.2006

12. Reuben DB, Walsh K, Moore AA, Damesyn M, Greendale GA. Hearing loss in community-dwelling older persons: National prevalence data and identification using simple questions. J Am Geriatr Soc. 1998;46(8):1008-11. [PMID: 9706892]

13. Agrawal Y, Platz EA, Niparko JK. Prevalence of hearing loss and differences by demographic characteristics among US adults: Data from the National Health and Nutrition Examination Survey, 1999-2004. Arch Intern Med. 2008; 168(14):1522-30. [PMID: 18663164]

DOI:10.1001/archinte.168.14.1522

14. Ventry IM, Weinstein BE. Identification of elderly people with hearing problems. ASHA. 1983;25(7):37-42. [PMID: 6626295]

15. Veterans Benefits Administration. Federal Regulations Book B, Supplement No. 72; Title 38, Part 3, Adjudication 55 FR 12349, Apr 3, 1990, as amended at 59 FR 60560, Nov 25, 1994. Washington (DC): Veterans Benefits Administration; 2006.

16. Ries PW. Prevalence and characteristics of persons with hearing trouble: United States, 1990-91. Vital Health Stat 10. 1994;(188):1-75. [PMID: 8165784$]$

17. Adams PF, Hendershot GE, Marano MA. Current estimates from the National Health Interview Survey, 1996. Vital Health Stat 10. 1999;(200):1-203. [PMID: 15782448$]$
18. Brown SC. Older Americans and tinnitus: A demographic study and chartbook. Washington (DC): Gallaudet Research Institute; 1990.

19. Hoffman HJ, Reed GW. Epidemiology of tinnitus. In: Snow JB, editor. Tinnitus: Theory and management. Hamilton, Ontario (Canada): BC Decker; 2004. p. 16-41.

20. Adams PF, Marano MA. Current estimates from the National Health Interview Survey, 1994. National Center for Health Statistics. Vital Health Stat 10. 1995;(193 Pt 1): 1-260. [PMID: 15789506]

21. Fausti SA, Wilmington DJ, Helt PV, Helt WJ, KonradMartin D. Hearing health and care: The need for improved hearing loss prevention and hearing conservation practices. J Rehabil Res Dev. 2005;42(4 Suppl 2):45-62.

[PMID: 16470464]

DOI:10.1682/JRRD.2005.02.0039

22. Saunders GH, Griest SE. Hearing loss in veterans and the need for hearing loss prevention programs. Noise Health. 2009;11(42):14-21. [PMID: 19265249]

DOI:10.4103/1463-1741.45308

23. Ewigman BG, Kivlahan CH, Hosokawa MC, Horman D. Efficacy of an intervention to promote use of hearing protection devices by firefighters. Pub Health Rep. 1990;105(1): 53-59. [PMID: 2106705]

24. Knobloch MJ, Broste SK. A hearing conservation program for Wisconsin youth working in agriculture. J Sch Health. 1998;68(8):313-18. [PMID: 9800180] DOI:10.1111/j.1746-1561.1998.tb00591.x

25. Lusk SL, Hong OS, Ronis DL, Eakin BL, Kerr MJ, Early MR. Effectiveness of an intervention to increase construction workers' use of hearing protection. Hum Factors. 1999;41(3):487-94. [PMID: 10665215] DOI:10.1518/001872099779610969

26. Lee-Feldstein A. Five-year follow-up study of hearing loss at several locations within a large automobile company. Am J Ind Med. 1993;24(1):42-54. [PMID: 8352292] DOI:10.1002/ajim.4700240105

27. Davies HW, Teschke K, Kennedy SM, Hodgson MR, Demers PA. Occupational noise exposure and hearing protector use in Canadian lumber mills. J Occup Environ Hyg. 2009; 6(1):32-41. [PMID: 18989837] DOI:10.1080/15459620802548940

28. Grotto J, Jones T. VA laboring under surge of wounded veterans. Chicago Tribune. 2010 Apr 1.

Submitted for publication August 13, 2010. Accepted in revised form November 22, 2010.

This article and any supplementary material should be cited as follows:

Folmer RL, McMillan GP, Austin DF, Henry JA. Audiometric thresholds and prevalence of tinnitus among male 
veterans in the United States: Data from the National Health and Nutrition Examination Survey, 1999-2006. J Rehabil Res Dev. 2011;48(5):503-16.
DOI:10.1682/JRRD.2010.07.0138

ResearcherID: Robert L. Folmer, PhD: E-3105-2011 
